\title{
Mechanical devices for solving quadratic and cubic equations
}

\author{
By G. D. C. Stokes.
}

Quadratic Equations. The device is designed to give the numerical value of the numerically smaller root of the equation $x^{2}-p x+q=0$, the other root and the signs of the roots $\alpha, \beta$ being found thereafter from the relations $a+\beta=p, a \beta=q$.

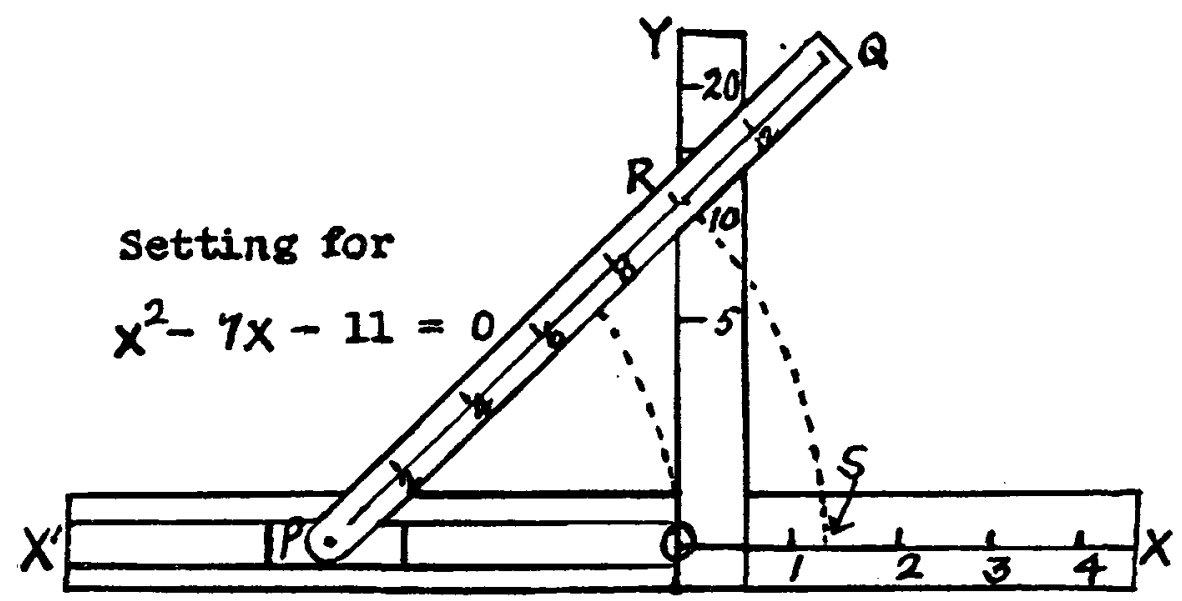

The apparatus consists of a base piece with central line $X^{\prime} O X$ carrying on $O X$ a uniform scale suitably graduated. An upright piece $O Y$ is fixed rigidly at right angles to the base piece and carries a scale marked " $y$ " at $R$, where $O R=\sqrt{ } y$. An arm $P Q$, made of transparent material, can rotate about the pivot $P$, which is attached to a slide moveable along $X^{\prime} O$, and carries a uniform scale whose unit is half that of the $O X$ scale.

The instrument is used in different ways according as $q$ is positive or negative. Consider as examples the equations $x^{2}-7 x+11=0$ (1) and $x^{2}-7 x-11=0(2)$. The numerical values of the smaller roots of these equations are $-\sqrt{ }\left\{\left(\frac{\frac{\pi}{2}}{2}\right)^{2}-11\right\}$ and $\sqrt{ }\left\{\left(\frac{\pi}{2}\right)^{2}+11\right\}-\frac{7}{2}$ respectively. In the case of (1), by moving the slide the mark " 7 " on $P Q$ is made to coincide with the reading " 11 " on $O Y$ at the point $R$. In (2), the reading " 7 " on $P Q$ is made to coincide with $O$, and $P Q$ is then rotated to pass through the point $R$ reading " 11 " on $O Y$. In both cases, $P R$ is then rotated into the position $P S$, and the numerical 
value of the smaller root of the equation is given by $O S$. The sign of this root and the remaining root of the equation can be readily determined from the coefficients in the equation.

If $p$ is negative, the equation can be transformed to the type considered by putting $x=-y$.

Also, if any reading should fall outside the limited scale of the model in use, the equation should first of all be transformed by putting $x=k y$ with a suitable value of $k$.

Cubic Equations. The device is in the form of a slide rule $A B$ with an arm $C D$ fixed at right angles to the stock in which the slide moves; a right-angled elbow piece $E F G$ is free to rotate about a pivot fixed at $F$ to a sliding head moveable along $C D$.

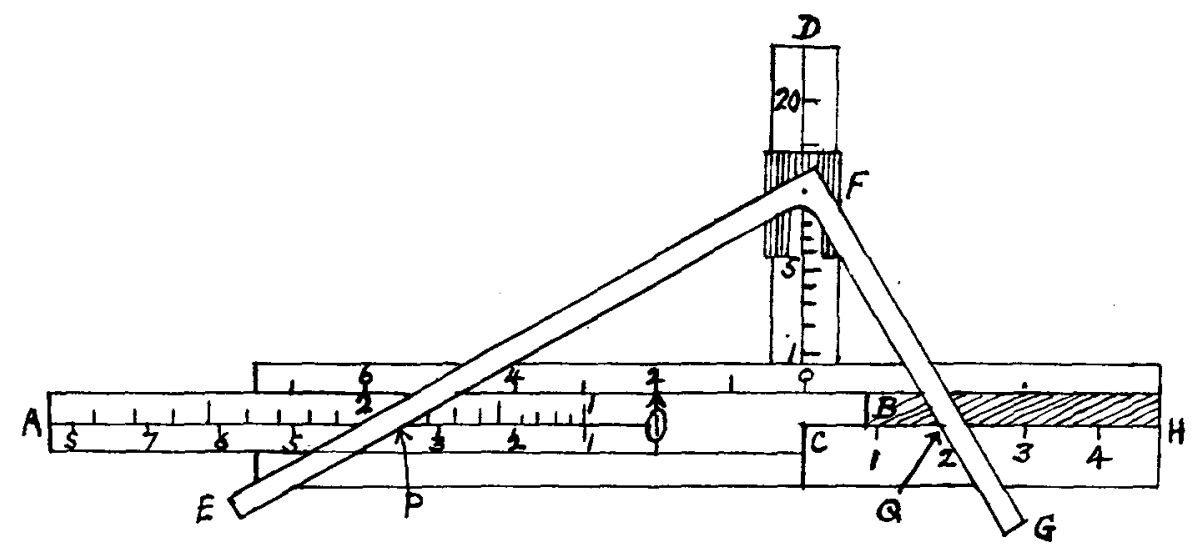

The equation to be solved must be in the reduced form $x^{3}+p x=q$.

$C D$ is graduated to a square root scale, so that when $F$ is set to the reading $q, C F=\sqrt{ } q(q=10$ in the diagram). The slide $A B$ is set to the reading $p(=O C=2$ in the diagram). $O A$ is graduated to a scale of squares, so that $O P=x^{2}$, when $x$ is the reading at $P$. A third scale $C H$, uniformly graduated, is fixed rigidly to the stock.

The device employs the theorem that, in the right angled triangle $P F Q, P C . C Q=C F^{2}$. Expressed algebraically, this gives $\left(x_{1}{ }^{2}+p\right) x_{2}=q$, where $x_{1}$ is the reading on the $x^{2}$ scale at $P$ and $x_{2}$ is the reading at $Q$. When $x_{1}=x_{2}$ a root of the equation is found; this can be effected by rotating the elbow piece $E F G$ about $F$ until the readings agree at $P$ and $Q$ (1.85 in the diagram). The root thus found is necessarily positive.

In the above it has been assumed that $q$ is positive. The various possibilities which may arise are as follows. 
(1) $p>0, q>0$. The equation has only one real root, which is positive and may be found as above.

(2) $p>0, q<0$. The equation has only one real root, which is negative. The root is found by transforming the equation by the substitution $x=-y$ and proceeding as above.

(3) $p<0, q>0$. The equation has one positive root which can be found as above and may have two negative roots. The number of real roots can be readily determined from a graph of the curve $(p / 3)^{3}+(q / 2)^{2}=0$, glued on to the back of the stock. As an example, consider the equation $x^{3}-6 x=3$. The positive root $x=2.67$ is found by the above procedure. Then, putting $x=-y$, the equation becomes $y\left(6-y^{2}\right)=3$, of which a positive root can be found as follows. Make $E F$ pass through the point $K$ reading $\sqrt{ } 6$ on $O A$ (so that $O K=6$ ) and adjust the slide until the readings at $C$ and $Q$ (where $F G$ crosses $C H$ ) are equal. This common reading gives the required root $y=2 \cdot 12$. Hence $x=2 \cdot 67$ and $x=-2.12$ are roots of the given equation. Since the sum of the roots is zero, the remaining root is $x=-0.55$.

(4) $p<0, q<0$. This case can be reduced to case (3) by putting $x=-y$.

If $q / p$ is very small, the accuracy of the instrument is low. However, since $x=q / p-x^{3} / p$, an approximation can be obtained by substituting $q / p$ for $x$ in the right hand side.

Finally, a uniform scale on $O A$ below the central line enables the instrument to be applied to the solution of the quadratic equation $x^{2}+p x=q$ in the same way.

Green Gates, 82 Greenock Road, LARGS. 